\title{
Inhaltsverzeichnis
}

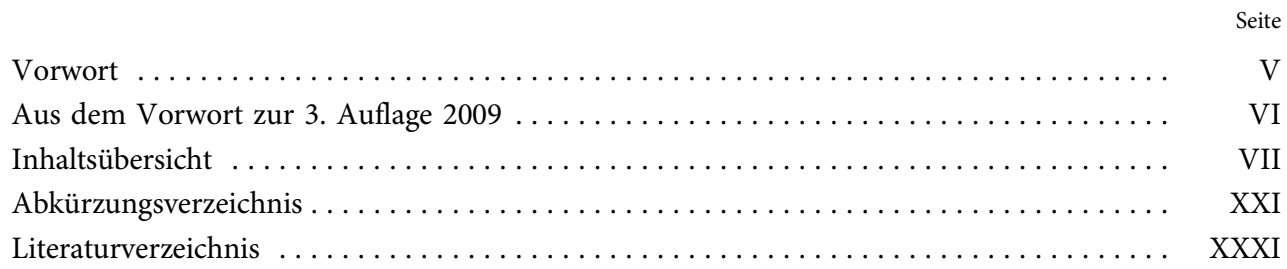

\section{Erster Teil: \\ Einführung}

$\$ 1$ Begriff und Struktur des Arbeitsrechts $\ldots \ldots \ldots \ldots \ldots \ldots \ldots \ldots \ldots \ldots \ldots \ldots$

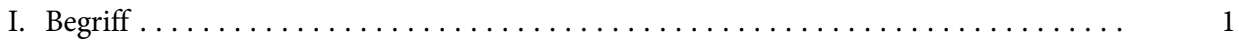

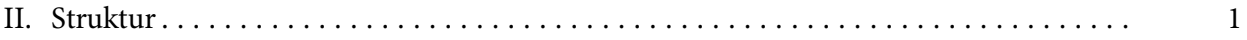

III. Stellung des Arbeitsrechts im Rechtssystem $\ldots \ldots \ldots \ldots \ldots \ldots \ldots \ldots \ldots \ldots \ldots \ldots . \ldots \ldots$

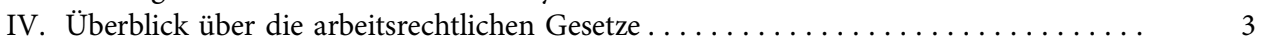

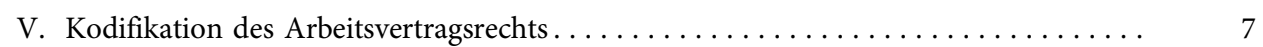

$\$ 2$ Überblick über die Literatur im Arbeitsrecht $\ldots \ldots \ldots \ldots \ldots \ldots \ldots \ldots \ldots \ldots$

I. Gesetzessammlungen $\ldots \ldots \ldots \ldots \ldots \ldots \ldots \ldots \ldots \ldots \ldots \ldots \ldots \ldots \ldots \ldots$

II. Lehrbücher $\ldots \ldots \ldots \ldots \ldots \ldots \ldots \ldots \ldots \ldots \ldots \ldots \ldots \ldots \ldots \ldots \ldots \ldots \ldots$

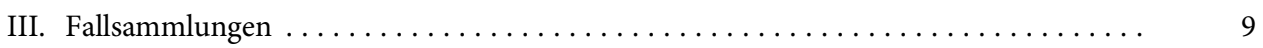

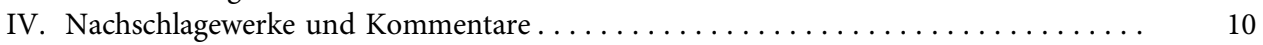

V. Entscheidungssammlungen $\ldots \ldots \ldots \ldots \ldots \ldots \ldots \ldots \ldots \ldots \ldots \ldots \ldots \ldots \ldots \ldots \ldots 11$

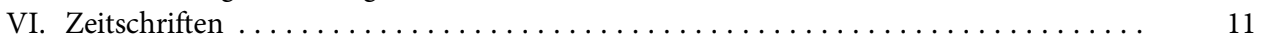

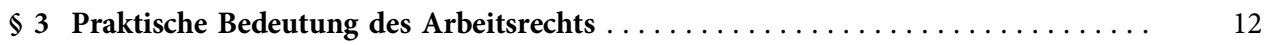

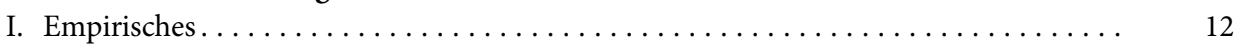

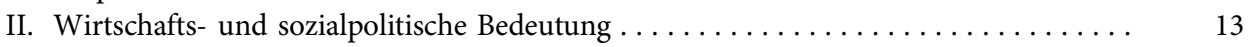

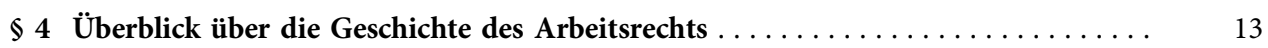

$\S 5$ Methodische Grundfragen des Arbeitsrechts $\ldots \ldots \ldots \ldots \ldots \ldots \ldots \ldots \ldots \ldots$

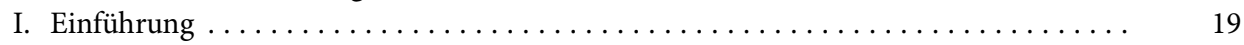

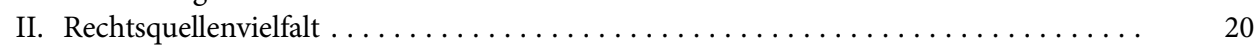

III. Auslegung partikularer und widersprüchlicher Gesetze $\ldots \ldots \ldots \ldots \ldots \ldots \ldots \ldots \ldots \quad 21$

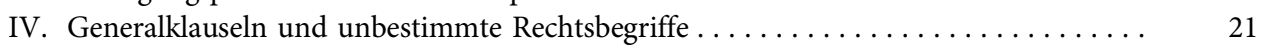

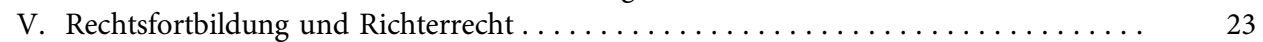

\section{Zweiter Teil:}

\section{Grundbegriffe des Arbeitsrechts}

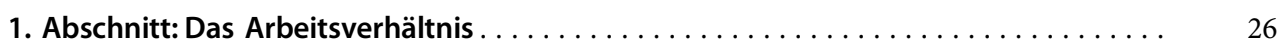

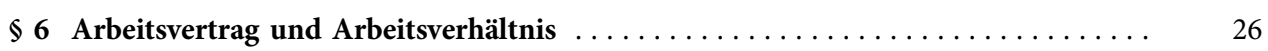

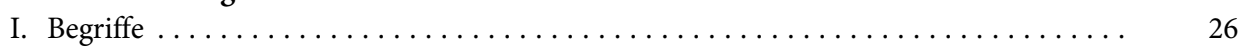

II. Abgrenzung zwischen Arbeitsvertrag und Arbeitsverhältnis . . . . . . . . . . 26 
Seite

III. Rechtsnatur des Arbeitsverhältnisses . . . . . . . . . . . . . . . . . . . . . 27

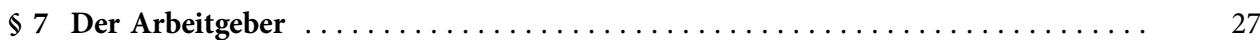

I. Begriff des Arbeitgebers $\ldots \ldots \ldots \ldots \ldots \ldots \ldots \ldots \ldots \ldots \ldots \ldots \ldots \ldots \ldots \ldots$

II. Voraussetzungen der Arbeitgebereigenschaft $\ldots \ldots \ldots \ldots \ldots \ldots \ldots \ldots \ldots \ldots \ldots \ldots$

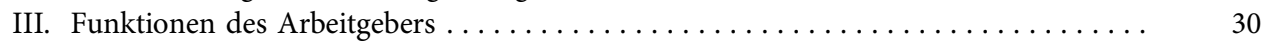

IV. Prozessuale Bedeutung der Arbeitgebereigenschaft $\ldots \ldots \ldots \ldots \ldots \ldots \ldots \ldots \ldots \ldots . \ldots \ldots$

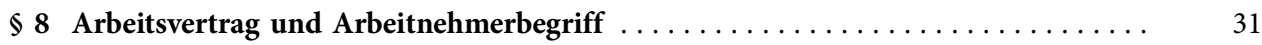

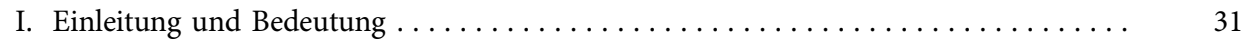

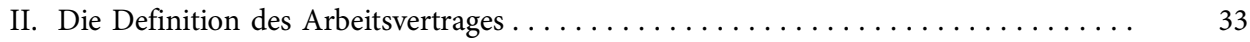

III. Vertiefungsproblem: Arbeitnehmer und Verbraucherschutzrecht . . . . . . . . 53

$\$ 9$ Besondere Arbeitnehmergruppen und arbeitnehmerähnliche Personen . . . . . . 57

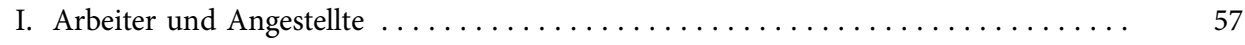

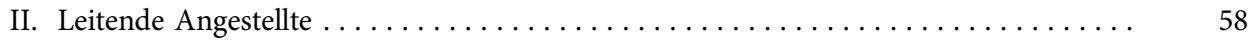

III. Arbeitnehmerähnliche Personen $\ldots \ldots \ldots \ldots \ldots \ldots \ldots \ldots \ldots \ldots \ldots \ldots \ldots \ldots$

IV. Zu ihrer Berufsbildung Beschäftigte $\ldots \ldots \ldots \ldots \ldots \ldots \ldots \ldots \ldots \ldots \ldots \ldots \ldots$

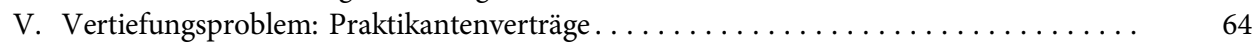

VI. Vertiefungsproblem: sog. Ein-Euro-Jobber $\ldots \ldots \ldots \ldots \ldots \ldots \ldots \ldots \ldots \ldots \ldots \ldots$

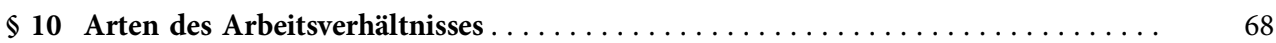

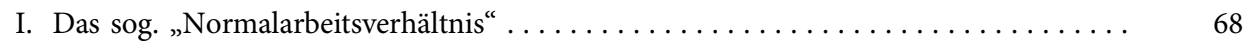

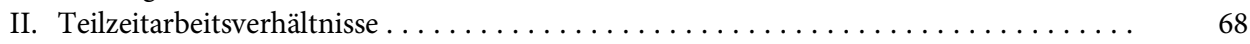

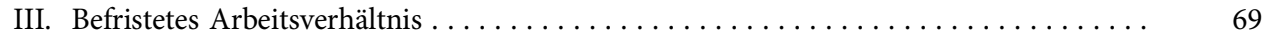

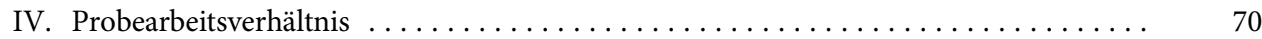

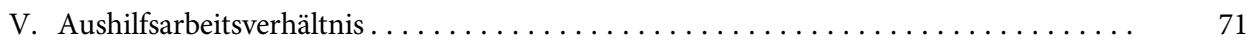

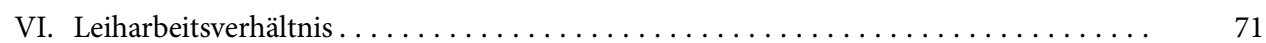

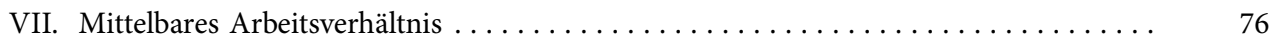

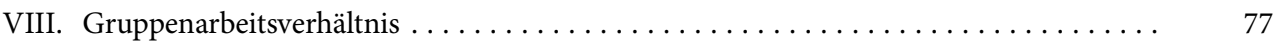

$\$ 11$ Bezugspunkte arbeitsrechtlicher Regelungen $\ldots \ldots \ldots \ldots \ldots \ldots \ldots \ldots \ldots \ldots$

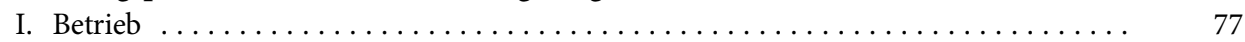

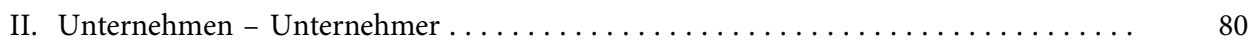

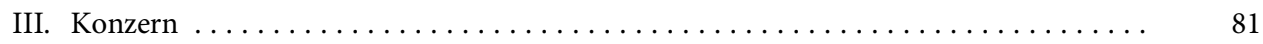

$\$ 12$ Interessenvertretungen von Arbeitnehmern und Arbeitgebern $\ldots \ldots \ldots \ldots \ldots \ldots$

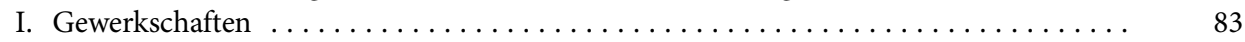

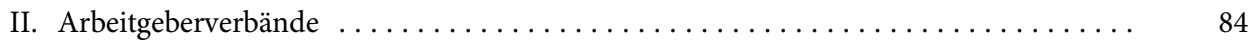

III. Aufgaben der Koalitionen $\ldots \ldots \ldots \ldots \ldots \ldots \ldots \ldots \ldots \ldots \ldots \ldots \ldots \ldots \ldots \ldots \ldots$

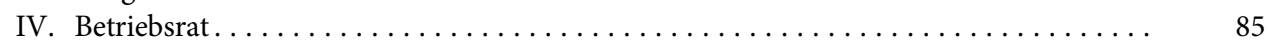

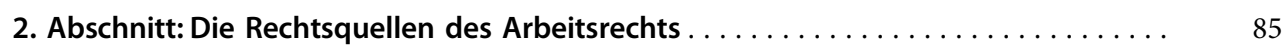

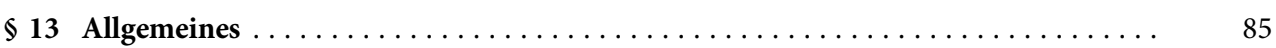

$\$ 14$ Supranationales und internationales Arbeitsrecht $\ldots \ldots \ldots \ldots \ldots \ldots \ldots \ldots \ldots$

I. Recht der Europäischen Union $\ldots \ldots \ldots \ldots \ldots \ldots \ldots \ldots \ldots \ldots \ldots \ldots \ldots$

II. Allgemeine völkerrechtliche Verträge $\ldots \ldots \ldots \ldots \ldots \ldots \ldots \ldots \ldots \ldots \ldots \ldots \ldots \ldots .114$

III. Internationales Privatrecht $\ldots \ldots \ldots \ldots \ldots \ldots \ldots \ldots \ldots \ldots \ldots \ldots \ldots \ldots \ldots \ldots$

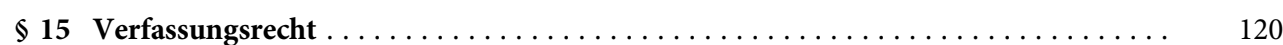

I. Bedeutung des Grundgesetzes für das Arbeitsrecht $\ldots \ldots \ldots \ldots \ldots \ldots \ldots \ldots \ldots \ldots \quad 120$

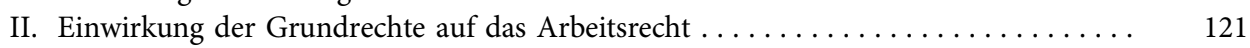

III. Einzelne Grundrechte . . . . . . . . . . . . . . . . . . . . . . . . . . . 128 
$\$ 16$ Gesetze und untergesetzliche Normen $\ldots \ldots \ldots \ldots \ldots \ldots \ldots \ldots \ldots \ldots \ldots \ldots$

I. Gesetze . . . . . . . . . . . . . . . . . . . . . . . . . . . . . . . . . . 149

II. Rechtsverordnungen $\ldots \ldots \ldots \ldots \ldots \ldots \ldots \ldots \ldots \ldots \ldots \ldots \ldots \ldots \ldots \ldots \ldots \ldots$

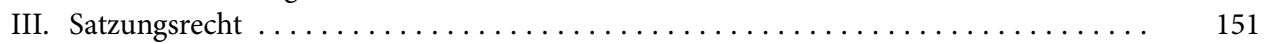

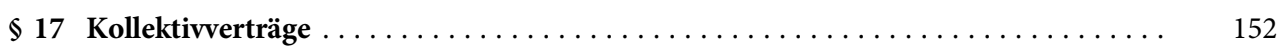

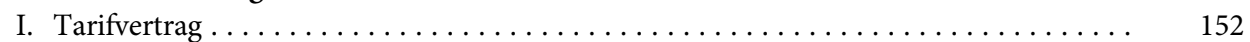

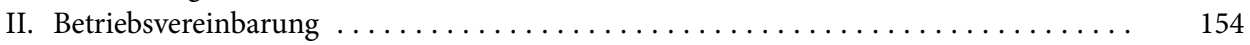

$\$ 18$ Regelungen auf arbeitsvertraglicher Ebene $\ldots \ldots \ldots \ldots \ldots \ldots \ldots \ldots \ldots \ldots \ldots$

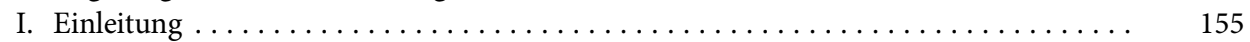

II. Einzelarbeitsvertrag $\ldots \ldots \ldots \ldots \ldots \ldots \ldots \ldots \ldots \ldots \ldots \ldots \ldots \ldots \ldots \ldots$

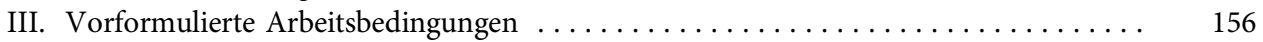

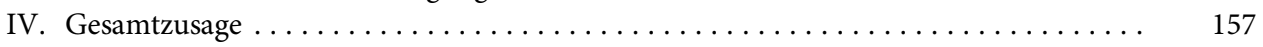

V. Konkludente Vertragsänderung („Betriebliche Übung“) . . . . . . . . . . . . . . 157

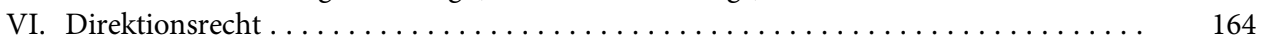

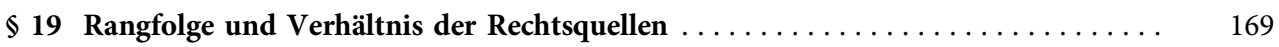

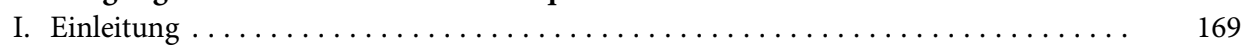

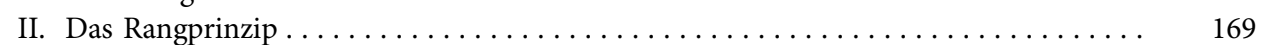

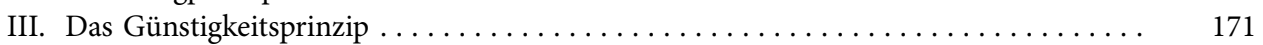

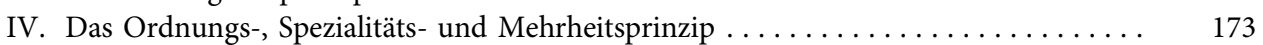

\section{Dritter Teil:}

\section{Begründung des Arbeitsverhältnisses}

$\$ 20$ Vertragsanbahnung $\ldots \ldots \ldots \ldots \ldots \ldots \ldots \ldots \ldots \ldots \ldots \ldots \ldots \ldots \ldots \ldots$

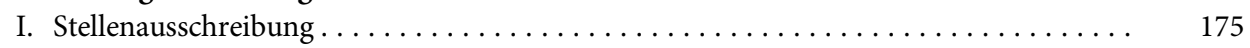

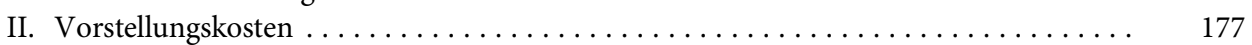

III. Fragerechte und Offenbarungspflichten $\ldots \ldots \ldots \ldots \ldots \ldots \ldots \ldots \ldots \ldots \ldots \ldots$

IV. Einstellungsuntersuchung und Einstellungstests $\ldots \ldots \ldots \ldots \ldots \ldots \ldots \ldots \ldots \ldots \ldots \quad 187$

V. Beteiligung des Betriebsrats ............................. 190

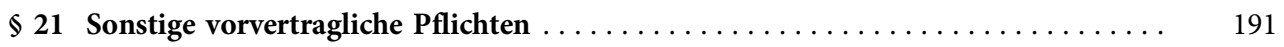

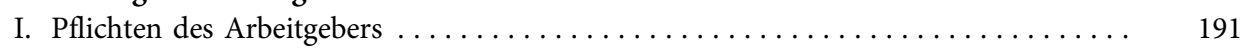

II. Pflichten des Arbeitnehmers $\ldots \ldots \ldots \ldots \ldots \ldots \ldots \ldots \ldots \ldots \ldots \ldots \ldots \ldots \ldots \ldots \ldots . . \ldots 192$

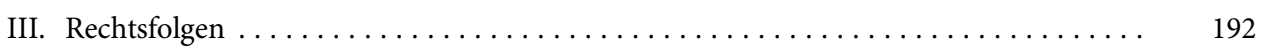

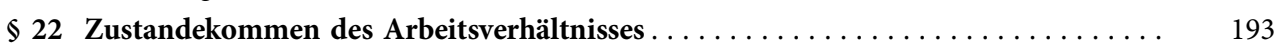

I. Abschluss des Arbeitsvertrags . . . . . . . . . . . . . . . . . . . . . . 193

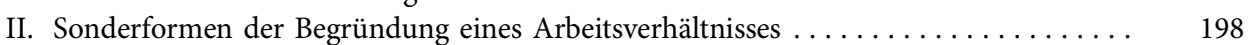

III. Abschlussfreiheit und Abschlussgebote . . . . . . . . . . . . . . . . . . . 199

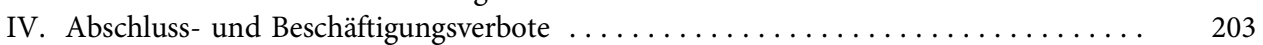

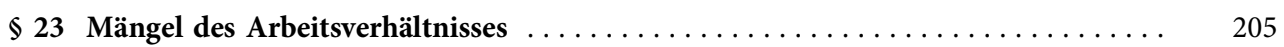

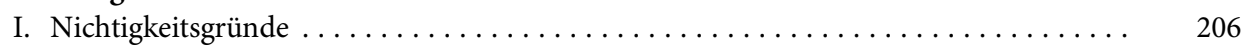

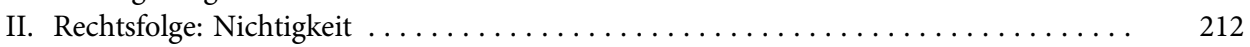




\section{Vierter Teil:}

\section{Inhalt des Arbeitsverhältnisses}

1. Abschnitt: Allgemeines $\ldots \ldots \ldots \ldots \ldots \ldots \ldots \ldots \ldots \ldots \ldots \ldots \ldots \ldots \ldots \ldots$

$\$ 24$ Der Arbeitsvertrag als gegenseitiger Vertrag $\ldots \ldots \ldots \ldots \ldots \ldots \ldots \ldots \ldots \ldots \ldots \ldots \ldots$

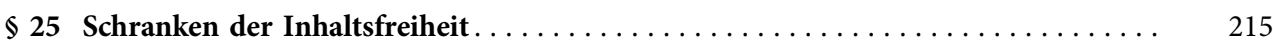

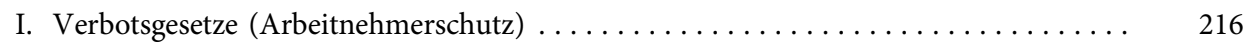

II. Verbot der Gesetzesumgehung $(\$ 134 \mathrm{BGB}) \ldots \ldots \ldots \ldots \ldots \ldots \ldots \ldots \ldots \ldots \ldots \ldots$

III. Verbot der Sittenwidrigkeit $(\$ 138$ BGB, $₫ 612 \mathrm{a}$ BGB $) \ldots \ldots \ldots \ldots \ldots \ldots \ldots \ldots \ldots$

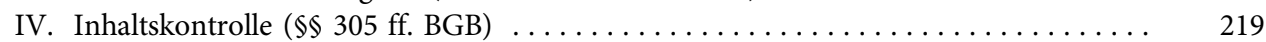

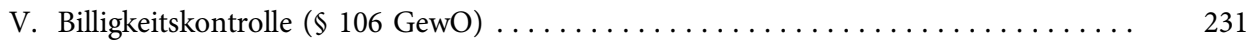

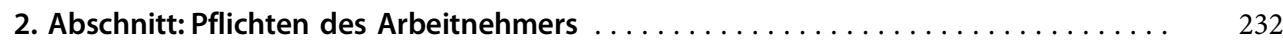

$\$ 26$ Hauptpflicht des Arbeitnehmers: Arbeitsleistung $\ldots \ldots \ldots \ldots \ldots \ldots \ldots \ldots \ldots$

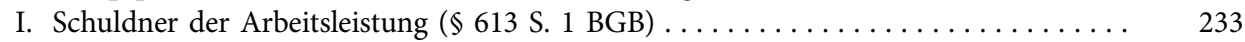

II. Gläubiger der Arbeitsleistung ( $\$ 613$ S. 2 BGB) $\ldots \ldots \ldots \ldots \ldots \ldots \ldots \ldots \ldots \ldots \ldots \ldots \ldots \ldots$

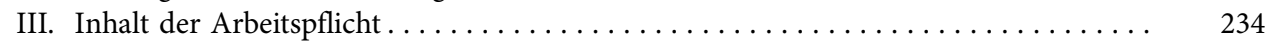

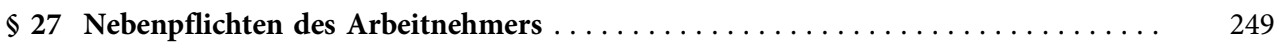

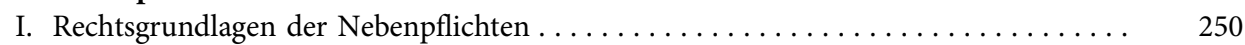

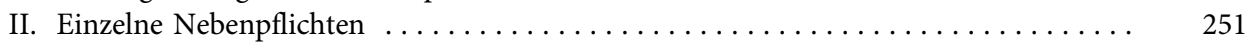

3. Abschnitt: Pflichten des Arbeitgebers $\ldots \ldots \ldots \ldots \ldots \ldots \ldots \ldots \ldots \ldots \ldots \ldots$

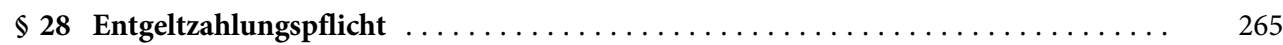

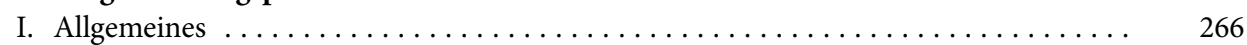

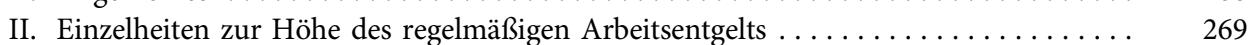

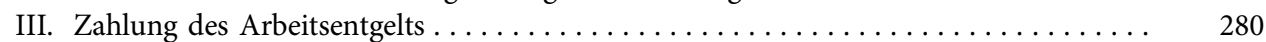

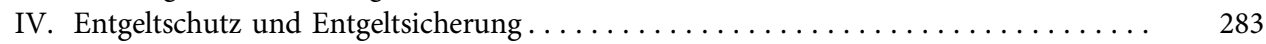

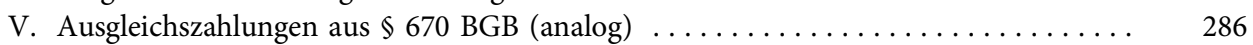

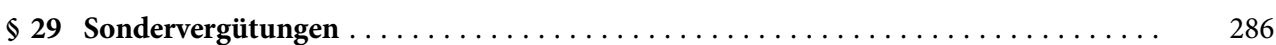

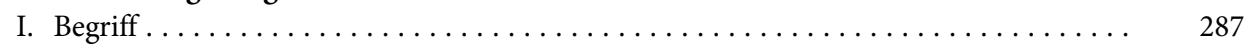

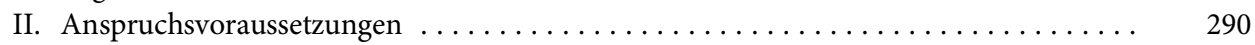

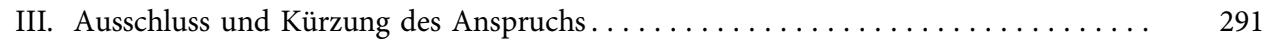

IV. Rückzahlungsklauseln . . . . . . . . . . . . . . . . . . . . . . . . . . . . . 295

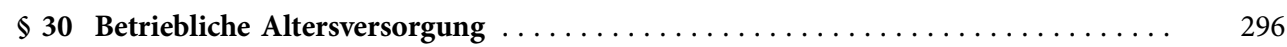

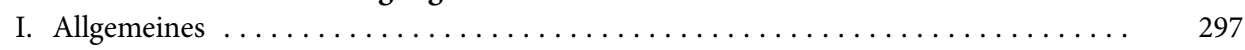

II. Widerruf von Versorgungsversprechen . . . . . . . . . . . . . . . . . . . . . . . . . . . . 299

III. Unverfallbarkeit, Insolvenzschutz und Rentenanpassung $\ldots \ldots \ldots \ldots \ldots \ldots \ldots \ldots$

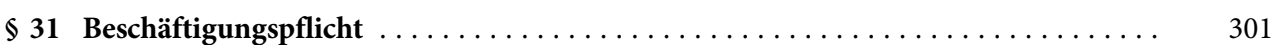

I. Rechtsgrundlagen der Beschäftigungspflicht $\ldots \ldots \ldots \ldots \ldots \ldots \ldots \ldots \ldots \ldots \ldots$

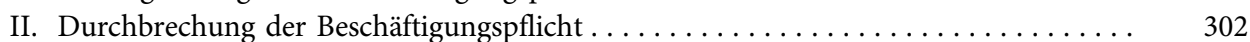

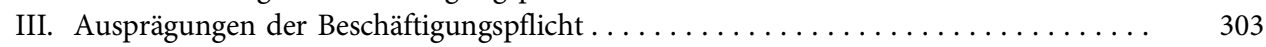

§ 32 Pflicht zur Gleichbehandlung und Antidiskriminierung: Überblick . . . . . . . . 304

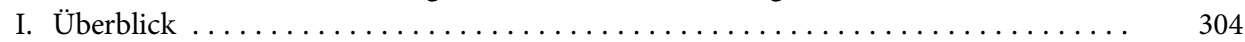

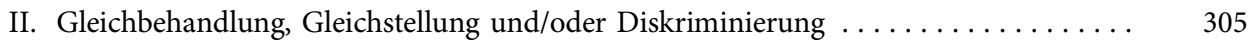

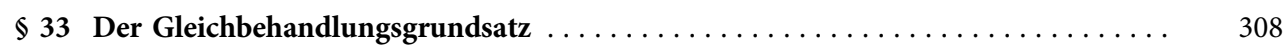

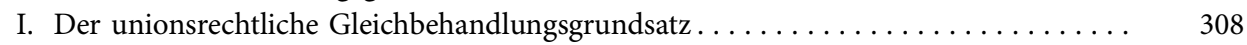


II. Der allgemeine arbeitsrechtliche Gleichbehandlungsgrundsatz . . . . . . . . . . . . . 309

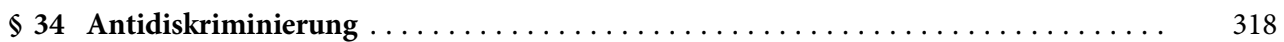

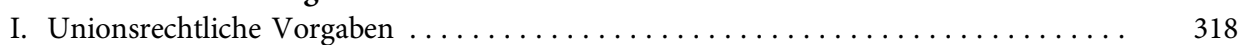

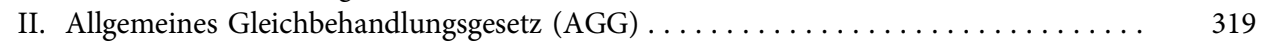

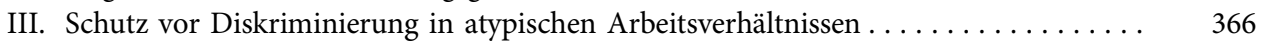

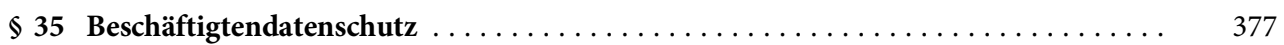

I. Das BDSG im Überblick . . . . . . . . . . . . . . . . . . . . . . . . . . . . . . . . 377

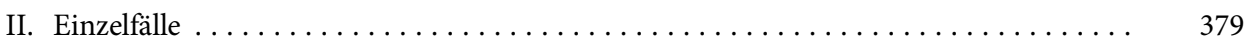

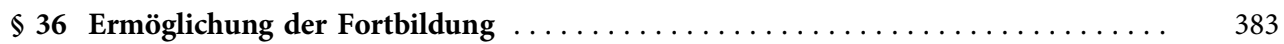

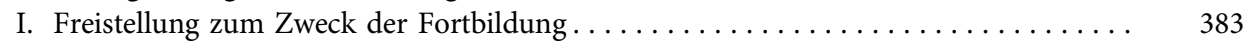

II. Rückzahlung von Fortbildungskosten $\ldots \ldots \ldots \ldots \ldots \ldots \ldots \ldots \ldots \ldots \ldots \ldots$

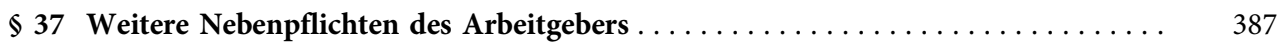

I. Grundlage der Arbeitgebernebenpflichten $\ldots \ldots \ldots \ldots \ldots \ldots \ldots \ldots \ldots \ldots \ldots \ldots \ldots \ldots$

II. Schutzpflichten des Arbeitgebers $\ldots \ldots \ldots \ldots \ldots \ldots \ldots \ldots \ldots \ldots \ldots \ldots \ldots \ldots \ldots \ldots$

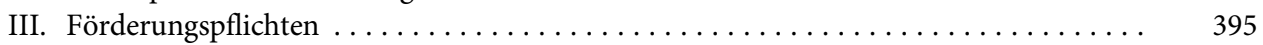

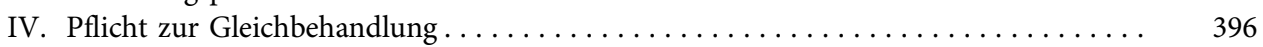

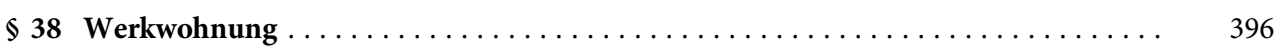

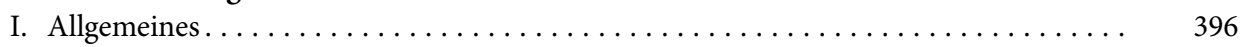

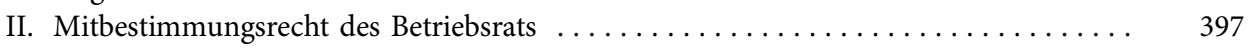

III. Die Rechtslage bei Werkmietwohnungen . . . . . . . . . . . . . . . . . . . . . . . 397

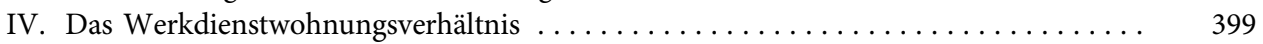

4. Abschnitt: Vertragsänderungen und Teilzeitarbeit $\ldots \ldots \ldots \ldots \ldots \ldots \ldots \ldots$

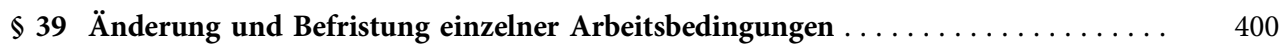

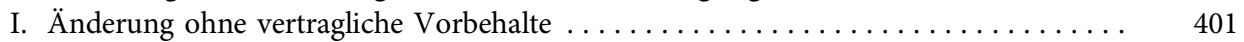

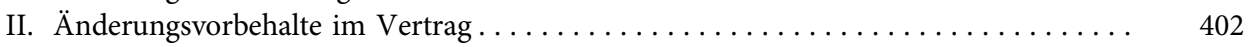

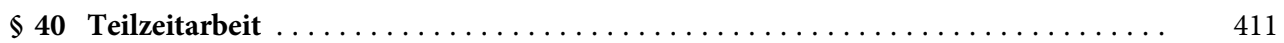

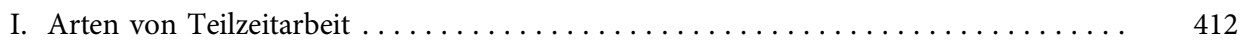

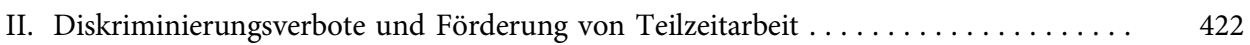

III. Rechtsanspruch auf Teilzeitarbeit $\ldots \ldots \ldots \ldots \ldots \ldots \ldots \ldots \ldots \ldots \ldots \ldots \ldots \ldots \quad 423$

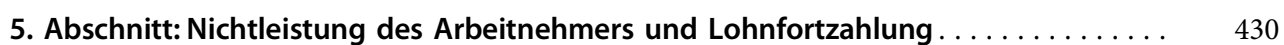

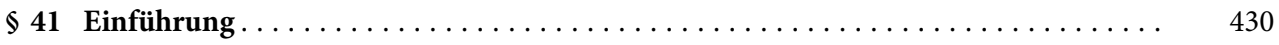

$\$ 42$ Lohnzahlung bei Nichtleistung der Arbeit $\ldots \ldots \ldots \ldots \ldots \ldots \ldots \ldots \ldots \ldots \ldots \ldots$

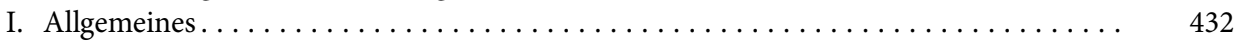

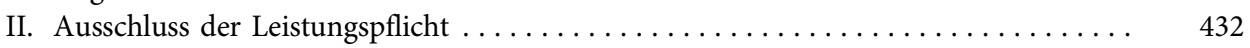

III. Rechtsfolgen des Ausschlusses der Leistungspflicht $\ldots \ldots \ldots \ldots \ldots \ldots \ldots \ldots$

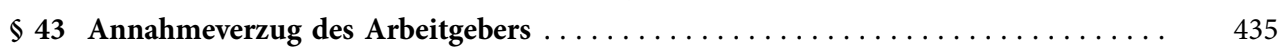

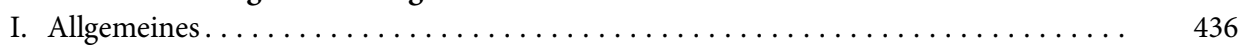

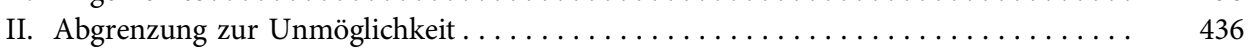

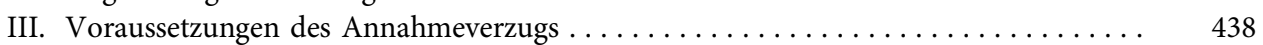

IV. Rechtsfolgen des Annahmeverzugs $\ldots \ldots \ldots \ldots \ldots \ldots \ldots \ldots \ldots \ldots \ldots \ldots \ldots 4$

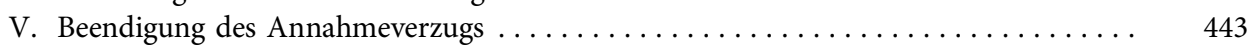

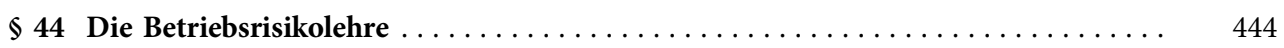

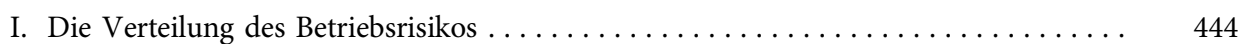

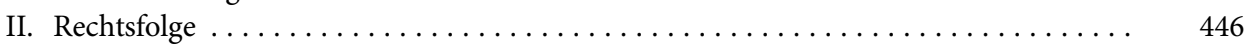


Seite

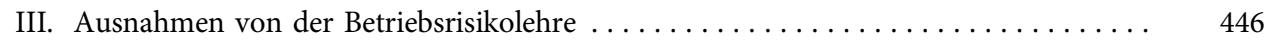

$\$ 45$ Arbeitsverhinderung aus persönlichen Gründen $(\$ 616$ BGB $) \ldots \ldots \ldots \ldots \ldots$

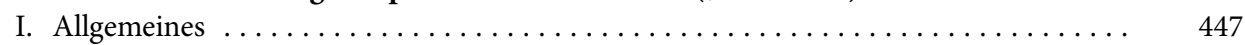

II. Anspruchsvoraussetzungen des $₫ 616$ BGB $\ldots \ldots \ldots \ldots \ldots \ldots \ldots \ldots \ldots \ldots \ldots 47$

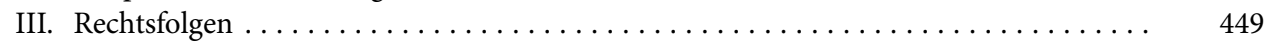

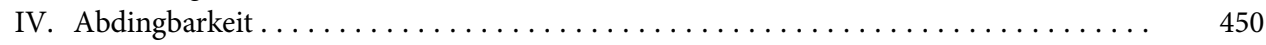

$\$ 46$ Entgeltfortzahlung im Krankheitsfall $\ldots \ldots \ldots \ldots \ldots \ldots \ldots \ldots \ldots \ldots \ldots$

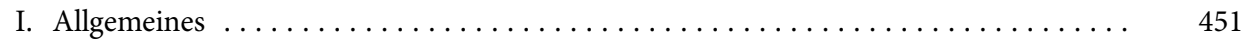

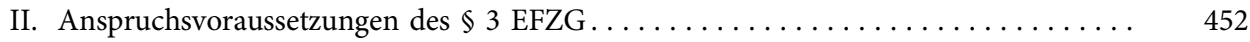

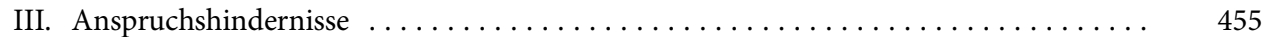

IV. Nachweis der Arbeitsunfähigkeit . . . . . . . . . . . . . . . . . . . . . . . 457

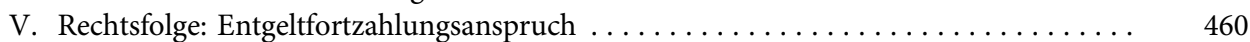

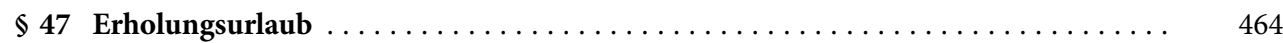

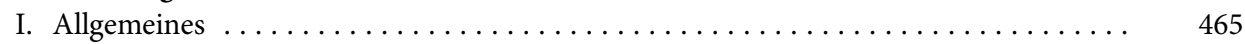

II. Anspruchsvoraussetzungen $\ldots \ldots \ldots \ldots \ldots \ldots \ldots \ldots \ldots \ldots \ldots \ldots \ldots \ldots \ldots \ldots$

III. Dauer des Erholungsurlaubs $\ldots \ldots \ldots \ldots \ldots \ldots \ldots \ldots \ldots \ldots \ldots \ldots \ldots \ldots \ldots$

IV. Erfüllung des Urlaubsanspruchs $\ldots \ldots \ldots \ldots \ldots \ldots \ldots \ldots \ldots \ldots \ldots \ldots \ldots \ldots \ldots$

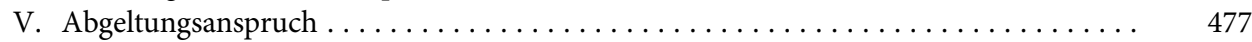

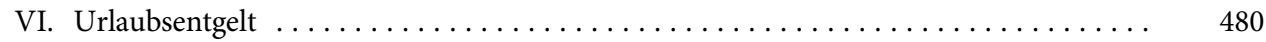

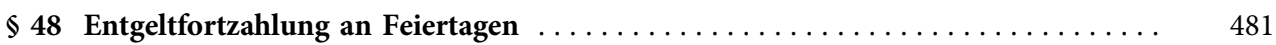

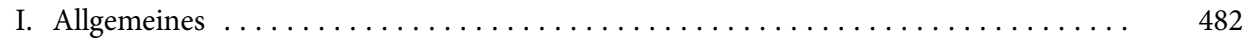

II. Anspruchsvoraussetzungen des $₫ 2$ Abs. 1 EFZG $\ldots \ldots \ldots \ldots \ldots \ldots \ldots \ldots \ldots . \ldots \ldots 2$

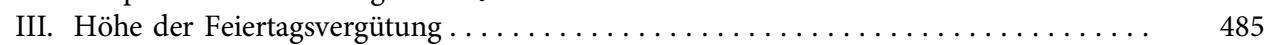

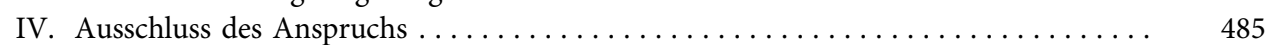

$\$ 49$ Mutterschutz $\ldots \ldots \ldots \ldots \ldots \ldots \ldots \ldots \ldots \ldots \ldots \ldots \ldots \ldots \ldots \ldots \ldots$

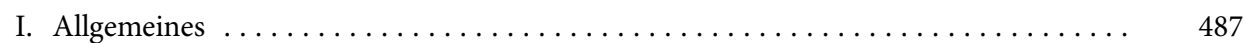

II. Die Regelungen des $\mathrm{MuSchG} \ldots \ldots \ldots \ldots \ldots \ldots \ldots \ldots \ldots \ldots \ldots \ldots$

$\$ 50$ Elterngeld, Elternzeit und Pflegezeit $\ldots \ldots \ldots \ldots \ldots \ldots \ldots \ldots \ldots \ldots \ldots \ldots \ldots$

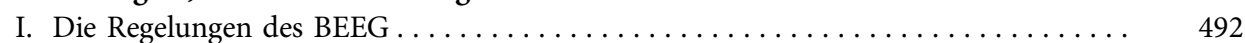

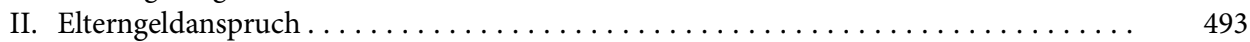

III. Elternzeit $\ldots \ldots \ldots \ldots \ldots \ldots \ldots \ldots \ldots \ldots \ldots \ldots \ldots \ldots \ldots \ldots \ldots \ldots \ldots \ldots \ldots$

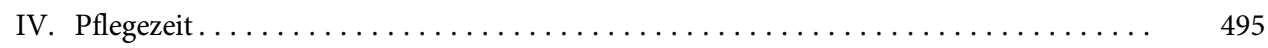

6. Abschnitt: Aufwendungsersatz und Schadensausgleich . . . . . . . . . . . . . 499

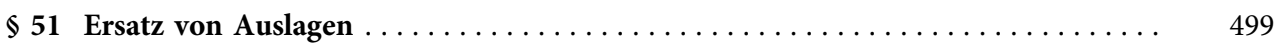

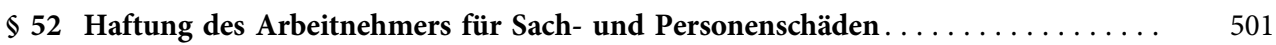

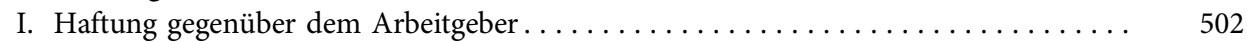

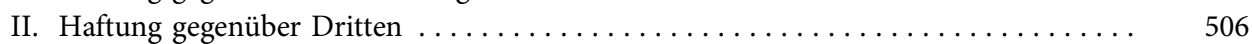

III. Mankohaftung ................................ 510

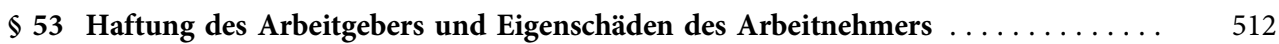

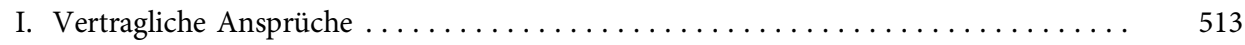

II. Deliktische Ansprüche und Gefährdungshaftung $\ldots \ldots \ldots \ldots \ldots \ldots \ldots \ldots \ldots$

III. Besonderheiten bei Personenschäden des Arbeitnehmers . . . . . . . . . . . . . 514

IV. Verschuldensunabhängiges Eintreten für Eigenschäden des Arbeitnehmers an Sachen 515 
7. Abschnite

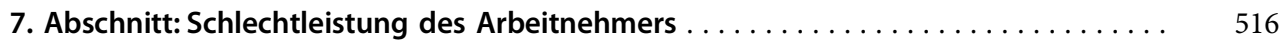

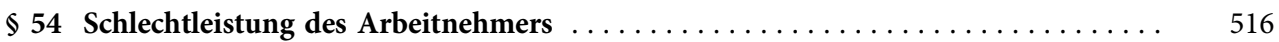

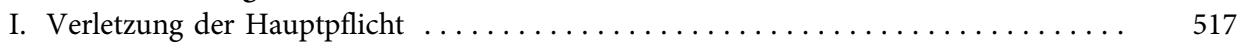

II. Verletzung von Nebenpflichten $\ldots \ldots \ldots \ldots \ldots \ldots \ldots \ldots \ldots \ldots \ldots \ldots \ldots \ldots \ldots \ldots \ldots$

\section{Fünfter Teil:}

\section{Beendigung des Arbeitsverhältnisses}

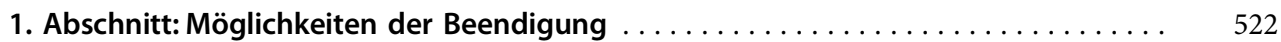

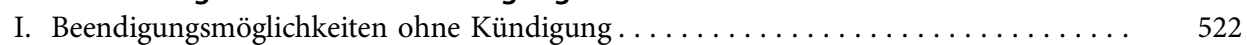

II. Abgrenzung zu anderen Rechtsinstituten und Maßnahmen . . . . . . . . . . 523

2. Abschnitt: Allgemeine Wirksamkeitsvoraussetzungen der Kündigung . . . . . . . 524

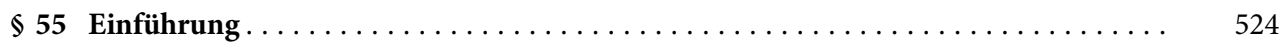

I. Der Bestandsschutz von Arbeitsverhältnissen in einer sozialen Marktwirtschaft . . . . 524

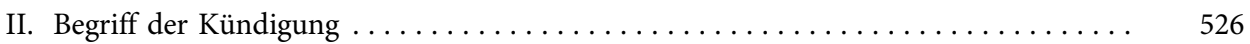

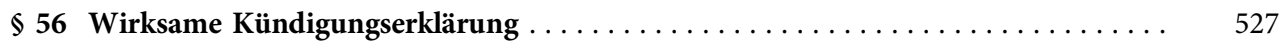

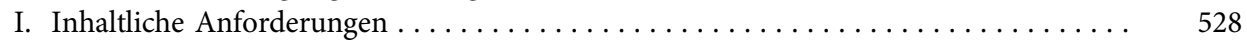

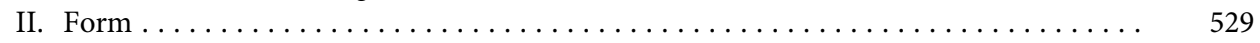

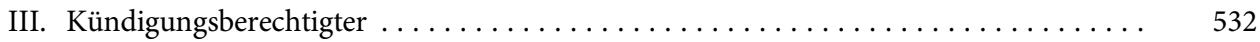

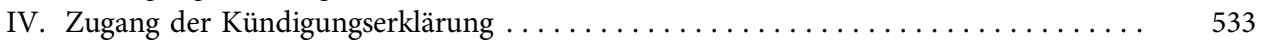

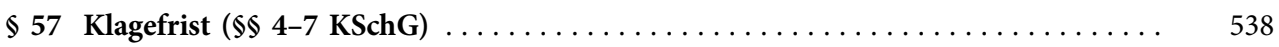

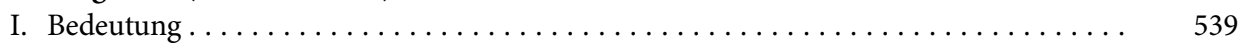

II. Eingreifen der Präklusion . . . . . . . . . . . . . . . . . . . . . . . . . 539

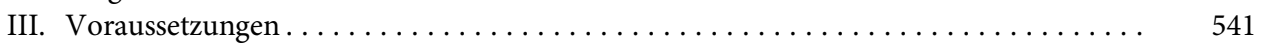

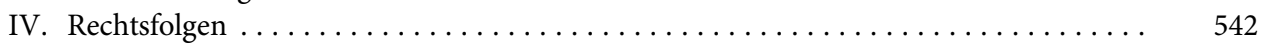

$\$ 58$ Allgemeine Unwirksamkeitsgründe und besondere Kündigungsverbote $\ldots \ldots \ldots .542$

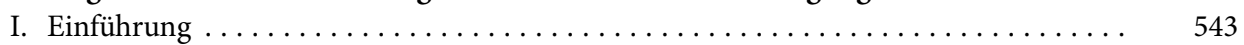

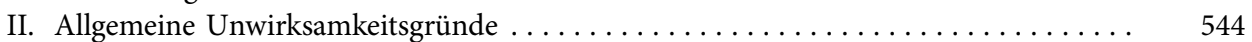

III. Gesetzliche Kündigungs- und Benachteiligungsverbote $\ldots \ldots \ldots \ldots \ldots \ldots \ldots \ldots \ldots$

IV. Präventive gesetzliche Kündigungsbeschränkungen $\ldots \ldots \ldots \ldots \ldots \ldots \ldots \ldots \ldots . \ldots \ldots 1$

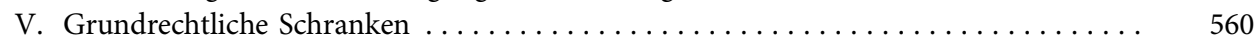

VI. Einzel- und tarifvertragliche Kündigungsbeschränkungen $\ldots \ldots \ldots \ldots \ldots \ldots \ldots \ldots . \ldots 61$

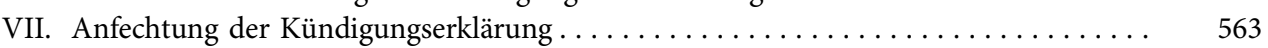

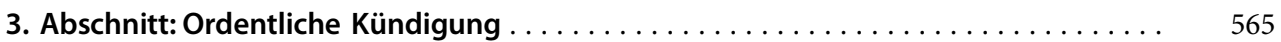

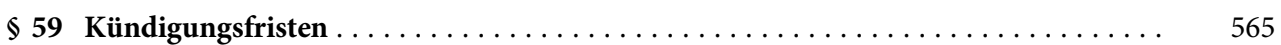

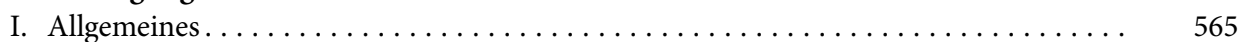

II. Gesetzliche Kündigungsfristen $\ldots \ldots \ldots \ldots \ldots \ldots \ldots \ldots \ldots \ldots \ldots \ldots \ldots \ldots \ldots$

III. Tarifliche Kündigungsfristen $\ldots \ldots \ldots \ldots \ldots \ldots \ldots \ldots \ldots \ldots \ldots \ldots \ldots \ldots \ldots \ldots$

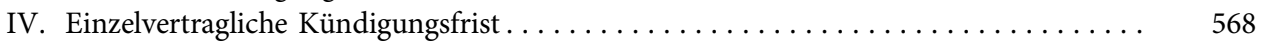

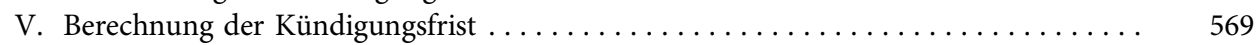

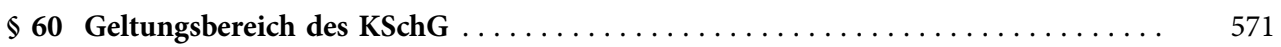

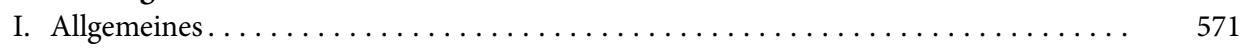

II. Arbeitnehmer . . . . . . . . . . . . . . . . . . . . . . . . . . 571 


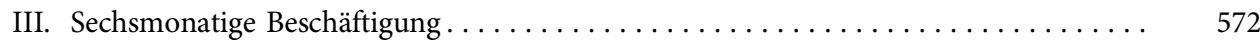

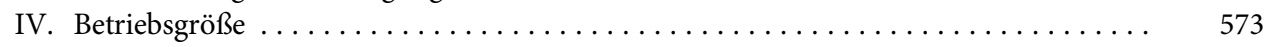

$\$ 61$ Sozialwidrigkeit der Kündigung - Allgemeines $\ldots \ldots \ldots \ldots \ldots \ldots \ldots \ldots \ldots \ldots$

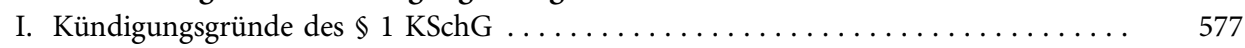

II. Beurteilungszeitpunkt; Prognoseprinzip $\ldots \ldots \ldots \ldots \ldots \ldots \ldots \ldots \ldots \ldots \ldots \ldots$

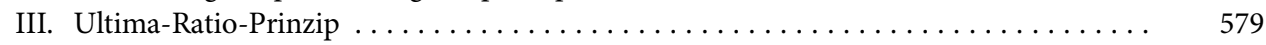

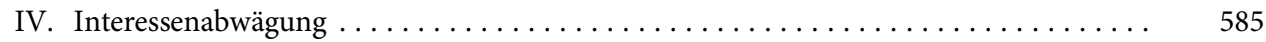

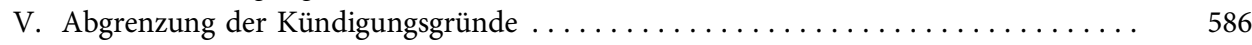

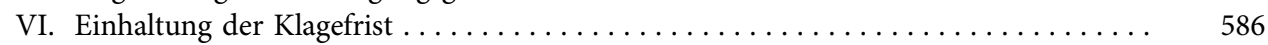

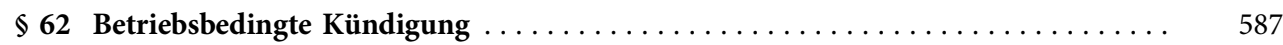

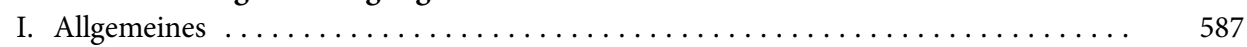

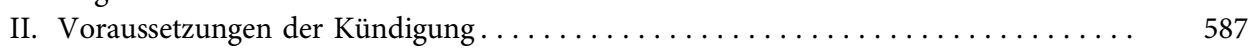

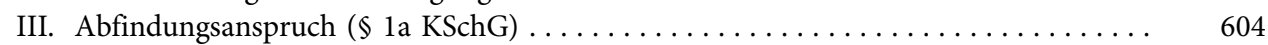

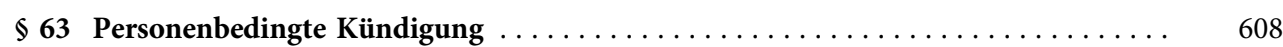

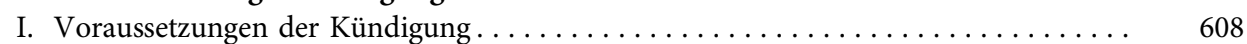

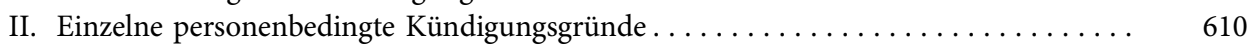

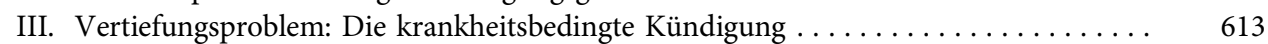

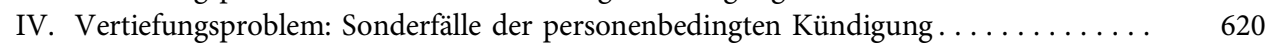

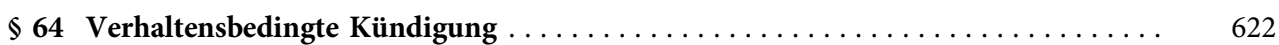

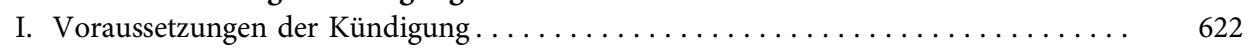

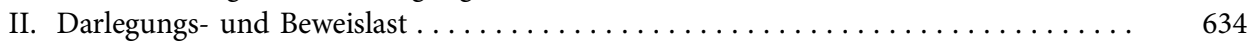

4. Abschnitt: Außerordentliche Kündigung $\ldots \ldots \ldots \ldots \ldots \ldots \ldots \ldots \ldots \ldots \ldots$

$\$ 65$ Voraussetzungen der außerordentlichen Kündigung $\ldots \ldots \ldots \ldots \ldots \ldots \ldots \ldots$

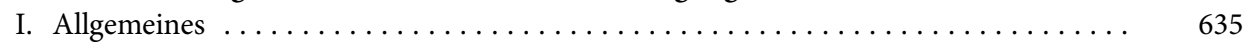

II. Allgemeine Wirksamkeitsvoraussetzungen $\ldots \ldots \ldots \ldots \ldots \ldots \ldots \ldots \ldots \ldots \ldots$

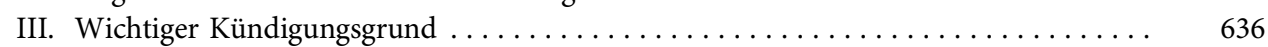

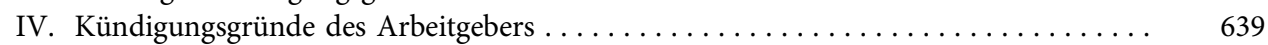

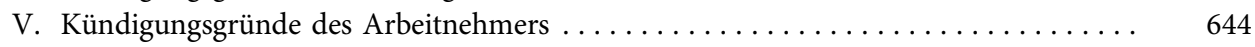

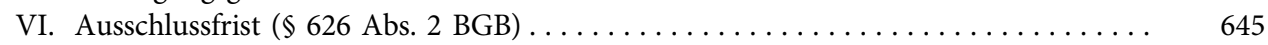

VII. Kündigung ordentlich unkündbarer Arbeitnehmer $\ldots \ldots \ldots \ldots \ldots \ldots \ldots \ldots \ldots .646$

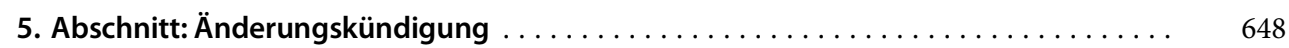

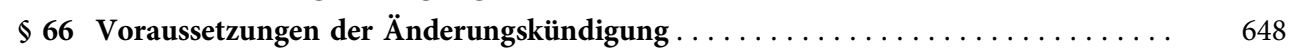

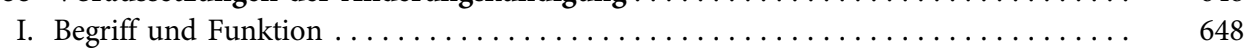

II. Reaktionsmöglichkeiten des Arbeitnehmers $\ldots \ldots \ldots \ldots \ldots \ldots \ldots \ldots \ldots \ldots \ldots \ldots .650$

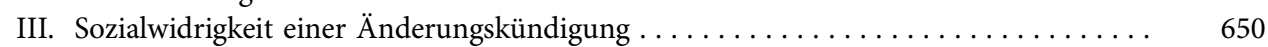

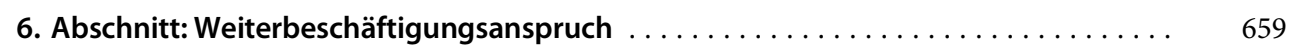

$\$ 67$ Besonderer und allgemeiner Weiterbeschäftigungsanspruch $\ldots \ldots \ldots \ldots \ldots \ldots$

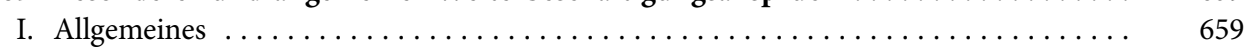

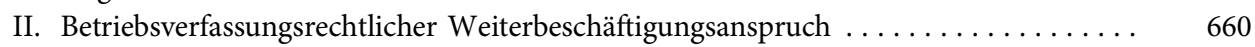

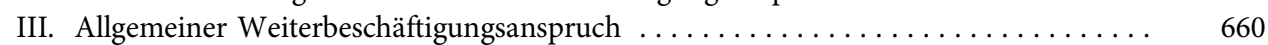


7. Abschnitt: Sonstige Beendigungstatbestände $\ldots \ldots \ldots \ldots \ldots \ldots \ldots \ldots \ldots \ldots \ldots \ldots$

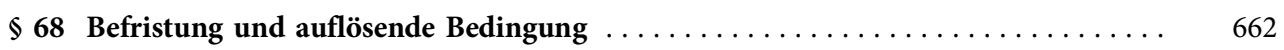

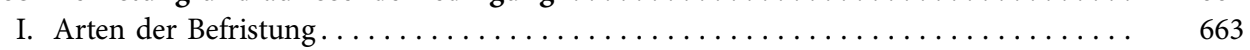

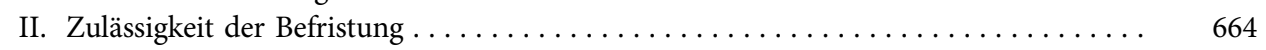

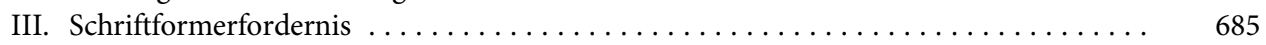

IV. Beendigung befristeter Arbeitsverhältnisse $\ldots \ldots \ldots \ldots \ldots \ldots \ldots \ldots \ldots \ldots \ldots \ldots \quad 688$

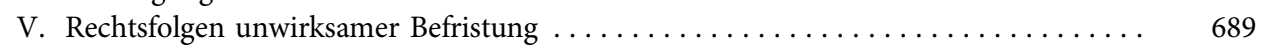

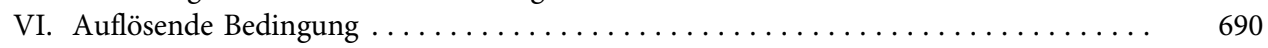

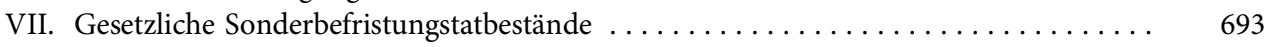

VIII. Gerichtliches Verfahren . . . . . . . . . . . . . . . . . . . . . . . . . . 696

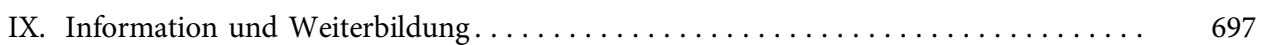

$\$ 69$ Weitere Beendigungstatbestände $\ldots \ldots \ldots \ldots \ldots \ldots \ldots \ldots \ldots \ldots \ldots \ldots \ldots$

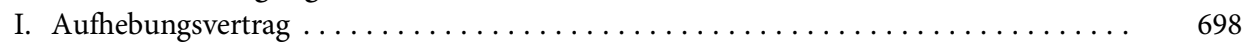

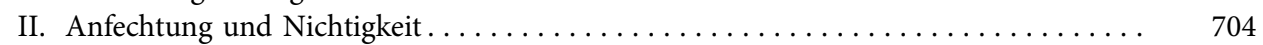

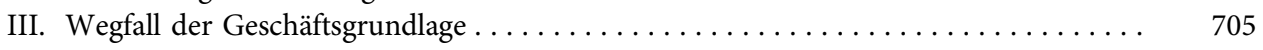

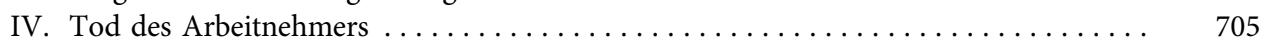

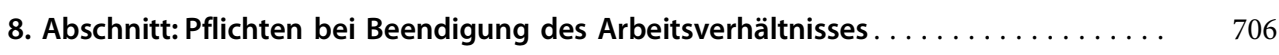

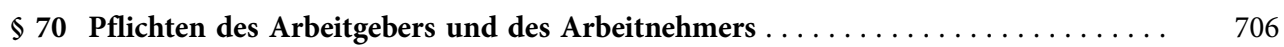

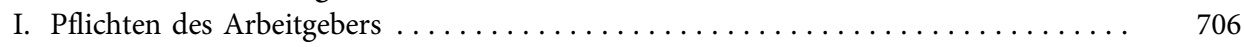

II. Pflichten des Arbeitnehmers $\ldots \ldots \ldots \ldots \ldots \ldots \ldots \ldots \ldots \ldots \ldots \ldots \ldots \ldots \ldots \ldots \ldots$

\section{Sechster Teil:}

\section{Wechsel des Betriebsinhabers}

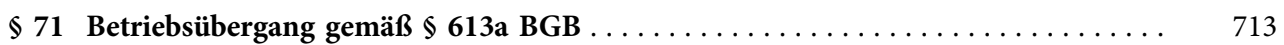

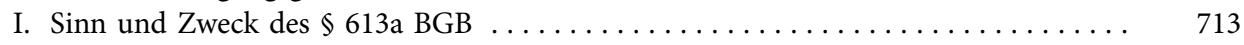

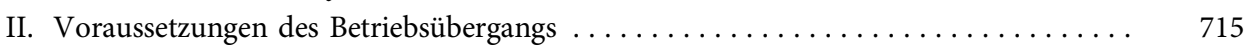

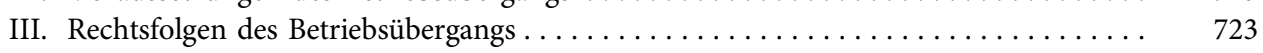

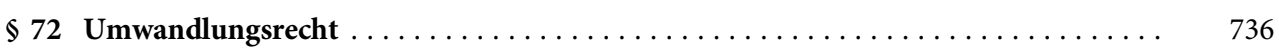

I. Regelungsgegenstand des Umwandlungsgesetzes . . . . . . . . . . . . . . 736

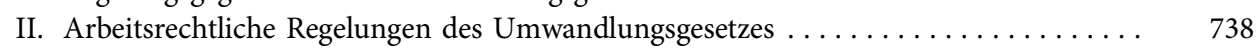

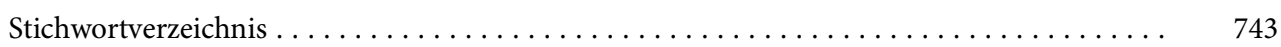


